\title{
Metamaterials Demonstrating Negative Thermal Capacity
}

\author{
Edward Bormashenko ${ }^{{ }_{1}}$, Evgeny Shulzinger \\ 1 Department of Chemical Engineering, Engineering Faculty, Ariel University, Ariel, Israel, 407000 \\ *Correspondance: $\quad$ edward@ariel.ac.il (E.B.)
}

\begin{abstract}
One-dimensional chain of core-shell pairs connected by ideal springs enables design of the metamaterial demonstrating the negative effective density and negative specific thermal capacity. We assume that the molar thermal capacity of the reported metamaterial is governed by the Dulong-Petit law in its high temperature limit. The specific thermal capacity depends of the density of the metamaterial; thus, it is expected to be negative, when the effective density of the chain is negative. The range of the frequencies enabling the effect of the negative thermal capacity is established. Dependence of the effective thermal capacity on the exciting frequency for various core/shell mass ratios is elucidated. The effective thermal capacity becomes negative in the vicinity of the local resonance frequency $\omega_{0}$ in the situation when the frequency $\omega$ approaches $\omega_{0}$ from above. The effect of the negative effective thermal capacity is expected in metals in the vicinity of the plasma frequency.
\end{abstract}

Keywords: metamaterials; negative effective mass; negative density; negative thermal capacity; resonance.

\section{Introduction}

21-st Century will demand for new materials and for the novel ideology of materials science, considering rapid depletion of natural resources and sustainability of the human society. Among the novel trends in the modern materials science the development of meta-materials are prominent [1-7]. Metamaterials are recently developed artificial materials demonstrating properties that are not found in naturally occurring materials. The domain of metamaterials covers a broad diversity of fields in physics and engineering, including: electromagnetics [3,8-9], acoustics [9-13], mechanics [5, 7] and thermodynamics [14]. In particular, metamaterials demonstrate negative values of refractive index $[1-3,8-9,11]$, bulk modulus $[11,15]$ and Poisson Ratio [16, 17].

Our paper is devoted to epy design of the metamaterial in which specific thermal capacity is expected to be negative. This effect becomes possible due to development of epy metamaterials possessing a negative effective density [18-26]. It was demonstrated that the dynamic effective mass (density) of an inhomogeneous mixture, used in the prediction of wave velocities in the long wavelength limit, can differ from the static version, namely the volume average of the component mass densities $[18,20,24,26]$. The effective mass density of certain kinds of elastic media built of mass-spring-shell oscillators becomes frequency dependent and may become negative for frequencies near the resonance frequency $[20,26]$. In our recent works we demonstrated that the effect of negative density may be achieved in the vicinity of frequency of plasma oscillations of electron gas embedded within the crystalline lattice [27-28]. Now, we demonstrate that the effect of negative density yields in a somewhat paradoxical way the negative effective thermal capacity solids. 


\section{Results and discussion}

Consider the 1D chain of oscillators presented in Figure 1 and treated in refs. [20, 25-28]. The 1D lattice, shown in Figure $\mathbf{1}$ is built from the core/shell oscillator pairs connected by elastic springs. A core mass $m_{2}$ is connected through the ideal spring with constant $k_{2}$ to a rigid shell with mass $m_{1}$. The system is excited by the harmonic force $F=\hat{F} \sin \omega t$. If we solve the Newtonian equations of motion for the masses $m_{1}$ and $m_{2}$ and replace the core/shell oscillator with a single effective mass $m_{\text {eff }}$ we derive Equation 1:

$$
m_{e f f}=m_{1}+\frac{m_{2} \omega_{0}^{2}}{\omega_{0}^{2}-\omega^{2}}
$$

where $\omega_{0}=\sqrt{\frac{k_{2}}{m_{2}}}$. It is immediately seen from Eq. 1 that when the frequency $\omega$ approaches $\omega_{0}$ from above the effective mass $m_{\text {eff }}$ will be negative [20,25-28]. The extension of the aforementioned approach to continuous medium leads to the idea of the effective negative density [25-26]. The effective density of the chain depicted in Figure $1 \rho_{\text {eff }}(\omega)$, was calculated in ref 26; and it is given by Eq. 2:

$$
\rho_{e f f}(\omega)=\rho_{s t} \frac{\theta}{\delta(1+\theta)\left(\frac{\omega}{\omega_{0}}\right)^{2}}\left\{\cos ^{-1}\left\{1-\frac{\delta}{2 \theta} \frac{\left(\frac{\omega}{\omega_{0}}\right)^{2}\left[\left(\frac{\omega}{\omega_{0}}\right)^{2}-(1+\theta)\right]}{\left(\frac{\omega}{\omega_{0}}\right)^{2}-1}\right\}\right\}^{2}
$$

where the static linear density of the chain $\rho_{s t}$ is given by: $\rho_{s t}=\frac{m_{1}+m_{2}}{a} ;\left[\rho_{s t}\right]=\frac{\mathrm{kg}}{\mathrm{m}}$ and $\theta=\frac{m_{2}}{\mathrm{~m}_{1}} ; \delta=$ $\frac{k_{2}}{k_{1}}$; and $a$ is the lattice constant (see Figure 1). The realization of the elastic medium with the negative effective density exploiting strong Mie-type resonance was suggested in ref. 25. Experimental realization of the elastic medium was the negative effective density was reported in ref. 29.

We demonstrate that the effective negative mass (density) media also give rise to the effect of negative effective thermal capacity. Consider 1D lattice of the core/shell pairs connected with ideal springs $k_{1}$, shown in Figure 1. Let us estimate the effective thermal capacity of this 1D lattice. In the limit of high temperatures the molar thermal capacity of the aforementioned chain (lattice) will be described by the modified Dulong-Petit equation [30-32]:

$$
c_{\text {mol }}=\text { const } \cong R
$$

where $c_{m o l}$ is the thermal capacity of the 1D ensemble built of Avogadro number of the core/shell oscillators, depicted in Figure 1, as established under the constant volume. The multiplier " 3 " is omitted in Eq. 3 due to the fact that we deal with the 1D chain of oscillators. It is noteworthy that the Dulong-Petit law works well in the realms of the both of classical and quantum mechanics, if the springs connecting the elements of lattice are supposed to be ideal [30-34]. The deviations from the Dulong-Petit law become essential when anharmonic effects are considered [35]; we restrict our treatment by the assumption that elastic springs are ideal. The effective dynamic specific thermal capacity $c_{e f f}(\omega)$ of the 1D medium, built of the oscillators, shown in Figure $\mathbf{1}$ is estimated as:

$$
c_{e f f}(\omega) \cong \frac{R}{\rho_{e f f(\omega)^{V} \text { mol }}},
$$


where $R$ is the gas constant, $V_{m o l}$ is the linear molar volume; $\left[V_{m o l}\right]=m ;\left[\rho_{\text {eff }}\right]=\frac{k g}{m}$ and $\left[c_{e f f}\right]=$ $\frac{J}{k g \times K}$. Combining Equations (2) and (4) immediately yields:

$$
c_{e f f}(\omega)=\frac{\delta(1+\theta)\left(\frac{\omega}{\omega_{0}}\right)^{2} R}{\rho_{s t} \theta V_{m o l}\left\{\cos ^{-1}\left\{1-\frac{\delta\left(\frac{\omega}{\omega_{0}}\right)^{2}\left[\left(\frac{\omega}{\omega_{0}}\right)^{2}-(1+\theta)\right]}{\left(\frac{\omega}{\omega_{0}}\right)^{2}-1}\right\}\right\}^{2}}
$$

Obviously, the field of frequencies in which $\rho_{\text {eff }}(\omega)<0$ takes place implies $c_{\text {eff }}(\omega)<0$. It is convenient to introduce the following designations: $\alpha=\frac{\delta(1+\theta) R}{\rho_{s t} \theta V_{m o l}}>0 ; \beta=\frac{\delta}{2 \theta}>0 ; \Omega=\frac{\omega}{\omega_{0}}>0$. Thus, Eq. 5 may be re-written as follows:

$$
c_{e f f}(\Omega)=\frac{\alpha \Omega^{2}}{\left\{\cos ^{-1}\left\{1-\beta \frac{\Omega^{2}\left[\Omega^{2}-(1+\theta)\right]}{\Omega^{2}-1}\right\}\right\}^{2}}
$$

The effective thermal capacity $c_{\text {eff }}(\Omega)$ will be complex, when Eq. 7 takes place:

$$
\frac{\Omega^{2}\left[\Omega^{2}-(1+\theta)\right]}{\Omega^{2}-1}<0
$$

Eq. 7 immediately yields:

$$
0<\Omega^{2}-1<\theta
$$

defining the range of frequencies at which the complex and in particular negative effective thermal capacity is expected. The dependencies $c_{e f f}(\Omega)$ are depicted in Figures 2-5. The MATLAB software was used for the preparing of the plots. We fixed the values of $\alpha$ and $\beta$, namely: $\alpha=1, \beta=1 / 2$, varied $\theta$ in the range $10^{-3}<\theta<10^{3}$ and plotted $c_{\text {eff }}(\Omega)$. Various configurations of the positive and negative branches of $c_{\text {eff }}(\Omega)$ are depicted in Figures 2-5. Consider, that generally the effective thermal capacity $c_{\text {eff }}(\Omega)$ is given by a complex number. We restrict our treatment within the range of frequencies supplying real values of effective thermal capacity (positive or negative), as shown in Figures 2-5. It should be emphasized that the critical frequency $\Omega_{c r}$ separating areas of positive and negative thermal capacities depends on the interrelation of masses $\theta=\frac{m_{2}}{m_{1}}$ as it recognized from Eq. 8 and Figures 2-5. The analytical expression for $\Omega_{c r}$ emerging from Eq. 6 is supplied by Eq. 9:

$$
\Omega_{c r}=\sqrt{1+\theta}
$$

When the core mass $m_{2}$ is markedly smaller than the shell mass $m_{1}$ (i.e. $\theta \ll 1$ ) the effective thermal capacity becomes negative in the vicinity of the local resonance frequency $\omega_{0}$, in the situation when the frequency $\omega$ approaches $\omega_{0}$ from above $\left(\Omega_{c r} \cong 1+\frac{\theta}{2}\right)$, as shown in Figure 2 . The case of the "light core mass" corresponds to the plasma oscillations of the free electron gas in metals, as discussed in detail in refs. 27-28 and depicted schematically in Figure 6. Thus, the effect of the negative effective thermal capacity is expected in metals in the vicinity of the plasma frequency.

It is instructive to establish the asymptotic behavior of Eq. 6, when $\theta \rightarrow 0 ; \beta \Omega^{2} \rightarrow 0$ takes place. The asymptotic is supplied by Eq. 10: 


$$
\lim _{\theta \rightarrow 0 ; \beta \Omega^{2} \rightarrow 0} c_{e f f}(\Omega)=\frac{\alpha \Omega^{2}}{\cos ^{-1}\left(1-\beta \Omega^{2}\right)} \cong-4 \alpha \beta \Omega^{2}
$$

It is recognized from Figures 2-5 that the effective thermal capacity $c_{e f f}(\Omega)$ demonstrates a behavior typical for the first order phase transitions, namely it exhibits discontinuity at $\Omega=\Omega_{c r}[30$ 32, 36]. However, this kind of the phase transition, may be called "pseudo-phase-transition" due the fact that the molar thermal capacity of the discussed lattice remains constant, and it is dictated by the Dulong-Petit rule.

What is the physical meaning of the "negative effective thermal capacity" ? It seems that it is absurd, which contradicts to the energy conservation. The close inspection of the problem shows, that the addressed core/shell lattice demonstrates the negative effective thermal capacity when exerted to the external harmonic vibrations; in other words it is excited by the external source of energy; thus, there is no conflict with the energy conservation. The more subtle problem is the analysis of the Second Law of Thermodynamics as applied for the addressed system, namely establishment of the direction of the energy flow for the chain built of core-shell oscillators in the vicinity of the internal resonance frequency. We plan to address this problem in our future investigations. We also plan to study the effect of the friction on the effective thermal capacity of the reported metamaterial.

\section{Conclusions}

We report one-dimensional metamaterials demonstrating the effective negative thermal capacity within the certain range of frequencies of the exciting force acting on the material. The metamaterial is built from the 1D chains comprising core/shell oscillators connected with the ideal springs. It was demonstrated that the effective density of such a chain if frequency-dependent and it may be negative, when the frequency of the external force approaches the resonance frequency from above [20, 25-28]. We adopt that the Dulong-Petit law is valid in the high temperature limit for the molar thermal capacity of the introduced metamaterial [30-34]. The specific effective thermal capacity $\left[c_{e f f}\right]=\frac{J}{k g \times K}$ depends on the effective dynamic density of the chain; and, thus, it depends on the frequency of the exciting force $c_{e f f}(\Omega)$ and it turns out to be complex for the certain range of dimensionless frequencies $\Omega=\frac{\omega}{\omega_{0}}$. The frequency dependence of the effective thermal capacity $\boldsymbol{c}_{\boldsymbol{e f f ( \boldsymbol { \Omega } )}}$ is influenced by the core/shell mass ratio $\theta=\frac{m_{2}}{m_{1}}$. The critical frequency $\boldsymbol{\Omega}_{\boldsymbol{c r}}$ separating areas of positive and negative thermal capacities is established as $\Omega_{c r}=\sqrt{1+\theta}$. The effective thermal capacity $c_{\text {eff }}(\Omega)$ demonstrates a behavior typical for the first order phase transitions, namely it exhibits discontinuity at $\Omega=\Omega_{c r}$ (recall that the molar thermal capacity of the metamaterial remains constant and it is governed by the Dulong-Petit law). The effect of the negative effective thermal capacity is predicted for metals in the vicinity of the electron gas plasma oscillations frequency, corresponding to: $\ll 1 ; \Omega_{c r} \cong 1+\frac{\theta}{2}$. The effect of the "negative effective thermal capacity does not conflict with the energy conservation. The investigated core/shell lattice demonstrates the negative 
effective thermal capacity when exerted to the external harmonic vibrations; in other words it is excited by the external source of energy; so there is no contradiction to the energy conservation law.

Acknowledgements: The authors are indebted to Mrs. Yelena Bormashenko for her kind help in preparing this work.

Author Contributions: Conceptualization, E.B., E.S.; methodology, E.B., E.S., software, E.S.; Validation, E. B., E.S.; formal analysis E. B., E.S.; investigation, E. B., E.S .; E.B.; writing-original draft preparation, E. B.; writing-review and editing; E.B., E.S.; supervision, E. B.; project administration, E.B.; funding acquisition, E.B. All authors have read and agreed to the submitted version of the manuscript

Funding: No external funding was obtained for this work.

Conflicts of Interest: The authors declare no conflict of interests.

\section{References}

1. Cui, T. J.; Smith, D.; Liu, R. Metamaterials. Theory, Design, and Applications, Springer, NY., USA, 2010.

2. Felbacq, D.; Bouchitté, G. Metamaterials Modelling and Design, Taylor \& Francis, Pan Stanford Publishing, Singapore, 2017.

3. Marques, R.; Martin, F.; Sorolla, M. Metamaterials with Negative Parameters: Theory, Design, and Microwave Applications (Wiley Series in Microwave and Optical Engineering Book 183), J. Wiley \& Sons, Hoboken, USA, 2008.

3. Engheta, N.; Ziolkowski, R. W. Electromagnetic Metamaterials: Physics and Engineering Explorations, IEEE Press, Hoes Lane, NJ, USA, 2006.

5. Wu, W.; Hu, W.; Qian, G.; Liao, H.; Xu, H.; Berto, F. Mechanical design and multifunctional applications of chiral mechanical metamaterials: A review. Materials \& Design 2019, 180, 107950.

6. Kshetrimayum, R. S. A Brief Intro to Metamaterials. IEEE Potentials. 2004, 23 (5), 44-46.

7. Bertoldi, K.; Vitelli, V.; Christensen, J. et al. Flexible mechanical metamaterials. Nat. Rev. Mater 2017, 2, 17066.

8. Boardman, A. D.; Grimalsky, V.V.; Kivshar, Y. S.; Koshevaya, S. V.; Lapine, M.; Litchinitser, N. M.; Malnev, V. N.; Noginov, M,; Rapoport, Y. G.; Shalaev, V. M. Active and tunable metamaterials. Laser \& Photonics Reviews 2011, 5 (2), 287-307.

9. Li, S. V.; Kivshar, Y. S..; Rybin, M. V. Toward Silicon-Based Metamaterials. ACS Photonics 2018, 5 (12), 47514757.

10. Kushwaha, M. S., Halevi, P., Dobrzynski, L.; \& Djafari-Rouhani, B. Acoustic band structure of periodic elastic composites. Phys. Rev. Lett. 1993, 71, 2022-2025.

11. Li, J.; Chan, C. T. Double-negative acoustic metamaterial. Phys. Rev. E 2004, 70, 055602(R).

12. Sheng, P.; Zhang, X. X.; Liu, Z.; Chan, C. T. Locally resonant sonic materials. Physica B 2003, 338 (1-4), 201205.

13. Liu, Zh.; Zhang, X.; Mao, Y.; Zhu, Y. Y.; Yang, Z.; Chan, C. T.; Sheng, P. Locally resonant sonic materials. Science 2000, 289 (5485), 1734-1736.

14. Maldovan, M. Phonon wave interference and thermal bandgap materials. Nature Mater 2015, 14, 667-674.

15. Ding, Y.; Liu, Zh.; Qiu, Ch.; Shi, J. Metamaterial with Simultaneously Negative Bulk Modulus and Mass Density. Phys. Rev. Lett. 2007, 99, 093904.

16. Babaee, S.; Shim, J.; Weaver, J. C.; Chen, E. R.; Patel, N.; Bertoldi, K. 3D Soft Metamaterials with Negative Poisson's Ratio. Adv. Mater. 2013, 25 (36), 5044-5049. 
17. Mizzi, L.; Azzopardi, K. M.; Attard, D.; Grima, J. N.; Gatt, R. Auxetic metamaterials exhibiting giant negative Poisson's ratios. Physica Status Solidi 2015, 9 (7), 425-430.

18. Berryman, J. G. Long-wavelength propagation in composite elastic media. I. Spherical inclusions. J. Acoust. Soc. Am. 1980, 680, 1809-1819.

19. Willis, J. R. 1985 The non-local influence of density variations in a composite. Int. J. Solids Struct. 1985, 210, 805-817.

20. Milton, G. W.; Willis, J. R. On modifications of Newton's second law and linear continuum elastodynamics. Proc. R. Soc. A 2007, 463, 855-880.

21. Liu, X.N.; Hu, G. K.; Huang, G. L.; Sun, C. T. An elastic metamaterial with simultaneously negative mass density and bulk modulus. Appl. Phys. Lett. 2011, 98, 251907.

22. Liu, Zh.; Zhang, X.; Mao, Y.; Zhu, Y. Y.; Yang, Z.; Chan, C. T.; Sheng, P. Locally resonant sonic materials. Science 2000, 289 (5485), 1734-1736.

23. Yang, Z.; Mei, J.; Yang, M.; Chan, N. H.; Sheng, P Membrane-type acoustic metamaterial with negative dynamic mass. Phys. Rev. Lett. 2008, 101, 204301.

24. Mei, J.; Liu, Z.; Wen, W.; Sheng, P. Effective mass density of fluid-solid composites. Phys. Rev. Lett. 2006, 96, 024301.

25. Chan, C. T.; Li, J.; Fung, K. H. On extending the concept of double negativity to acoustic waves. JZUS A 2006, 7, 24-28.

26. Huang, H. H.; Sun, C. T.; Huang, G. L. On the negative effective mass density in acoustic metamaterials. Int. J. Eng. Sci. 2009, 47, 610-617.

27. Bormashenko, Ed.; Legchenkova, I. Negative effective mass in plasmonic systems. Materials 2020, 13 (8), 1890. 28. Bormashenko, Ed; Legchenkova, I.; Frenkel, M. Negative Effective Mass in Plasmonic Systems II: Elucidating the Optical and Acoustical Branches of Vibrations and the Possibility of Anti-Resonance Propagation. Materials 2020, 13(16), 3512.

29. Yao, Sh.; Zhou, X.; Hu, G. Experimental study on negative effective mass in a 1D mass-spring system. New J. Physics 2008, 10, 043020.

30. Baierlein, R. Thermal Physics. Cambridge University Press, Cambridge, UK, 1999.

31. Landau, L. D.; Lifshitz, E.M. Statistical Physics, 3rd ed., Course of Theoretical Physics, Vol. 5. Elsevier, Oxford, UK, (2011).

32. Schroeder, D.V. Thermal Physics, Addison Wesley Longman, San Francisco, USA, 2000.

33. de Liano, M. Dulong-Petit law in a van der Waals theory of the crystalline state. Am. J. Physics 1981, 49, 489.

34. Khodel, V.A.; Clark, J.W.; Shaginyan, V.R.; Zverev, M.V. Second wind of the Dulong-Petit law at a quantum critical point. Jetp Lett. 2010, 92, 532-536.

35. Stern, E. A. Theory of the Anharmonic Properties of Solids. Phys. Rev. 1958, 111, 786.

36. Thiele, U.; Frohoff-Hülsmann, T.; Engelnkemper, S.; Knobloch, E.; Archer, A, J. First order phase transitions and the thermodynamic limit. New J. Physics 2019, 21, 123021. 


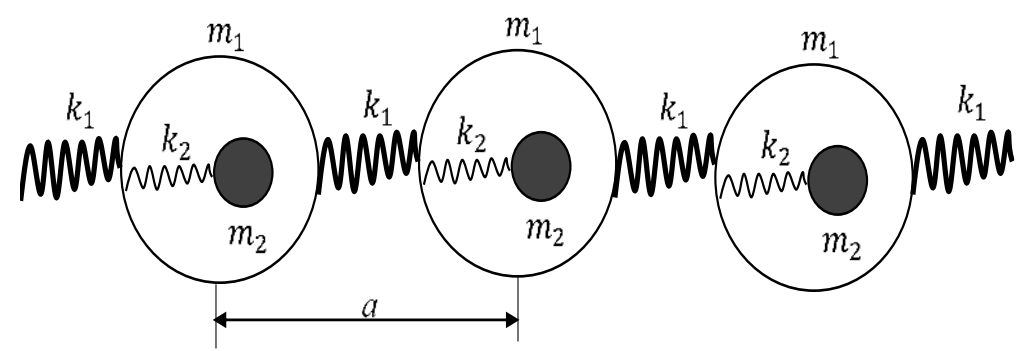

Figure 1. The chain of core/shell pairs connected by ideal springs $\boldsymbol{k}_{\mathbf{1}}$ make possible 1D medium possessing negative effective density and thermal capacity. 


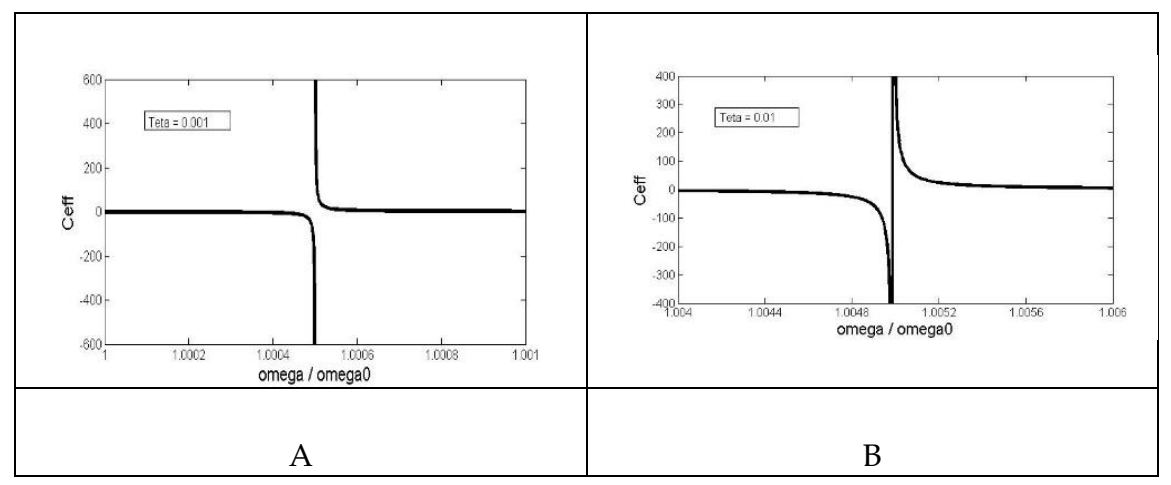

Figure 2. The dependence $\boldsymbol{c}_{\boldsymbol{e f f}}(\boldsymbol{\Omega}) ; \boldsymbol{\Omega}=\frac{\omega}{\omega_{0}}$ is depicted; A. $\boldsymbol{\theta}=\mathbf{0 . 0 0 1} ; \mathbf{B} . \boldsymbol{\theta}=\mathbf{0 . 0 1}$ 


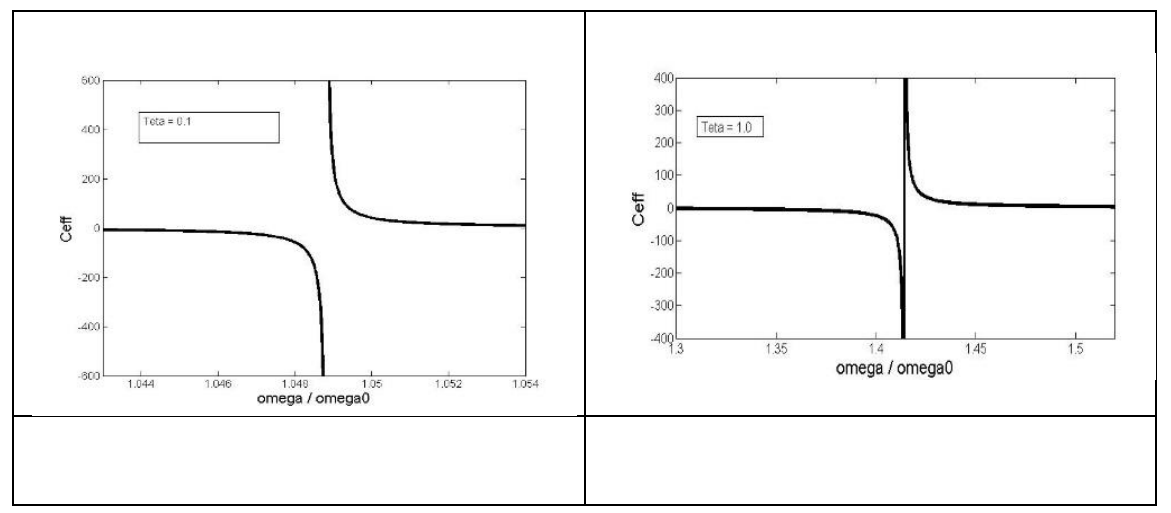

Figure 3. The dependence $c_{e f f}(\Omega) ; \Omega=\frac{\omega}{\omega_{0}}$ is depicted; A. $\theta=0.1 ;$ B. $\theta=1$. 


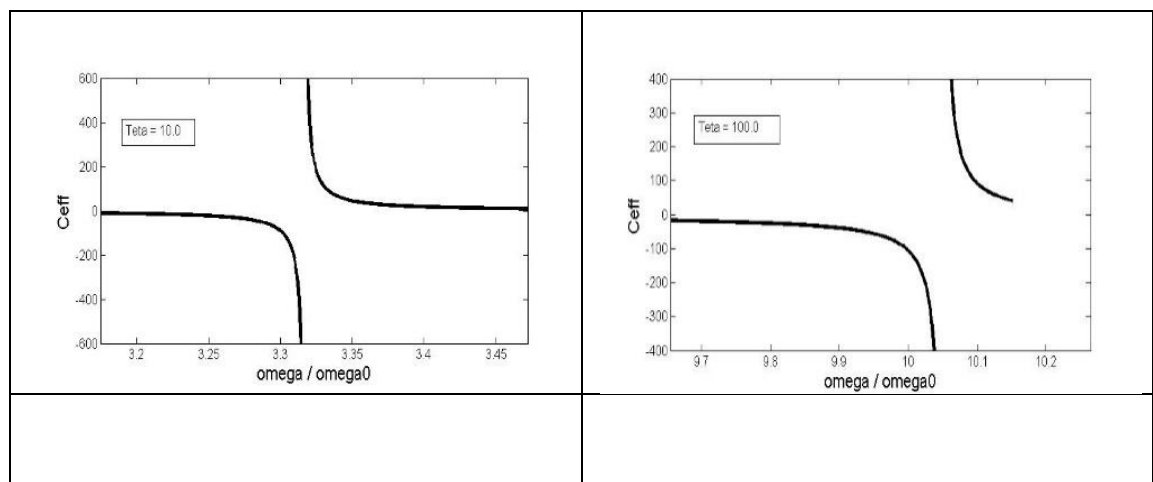

Figure 4. The dependence $c_{e f f}(\Omega) ; \Omega=\frac{\omega}{\omega_{0}}$ is depicted; A. $\theta=10 ;$ B. $\theta=100$. 


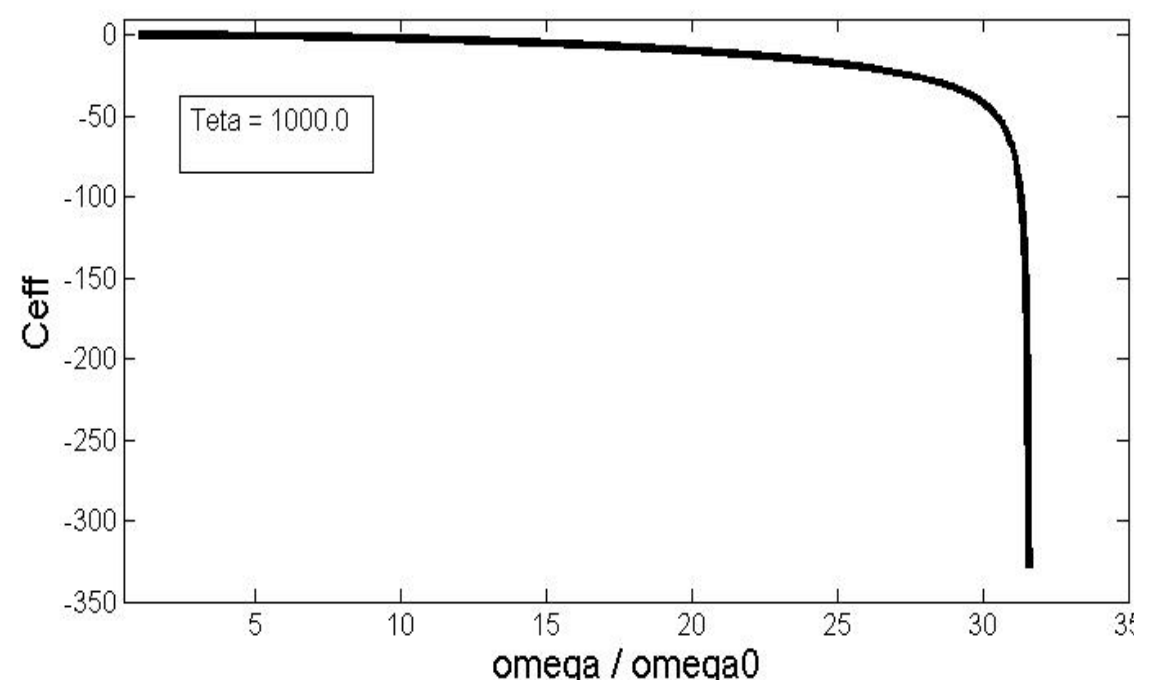

Figure 5. The dependence $c_{\text {eff }}(\Omega) ; \Omega=\frac{\omega}{\omega_{0}}$ is depicted; $\theta=1000$. 


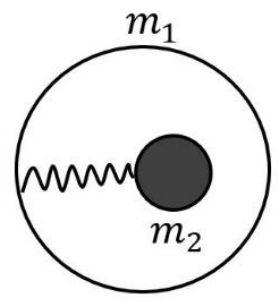

A

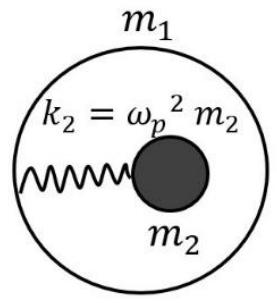

B

Figure 6. A. Core with mass $m_{2}$ is connected internally through the spring with $k_{2}$ to a shell with mass $m_{1}$. The system is subjected to the sinusoidal force $F(t)=\hat{F} \sin \omega t$. B. Free electrons gas $m_{2}$ is embedded into the ionic lattice $m_{1} ; \omega_{p}$ is the plasma frequency (the left sketch).

The equivalent mechanical scheme of the system (right sketch). 


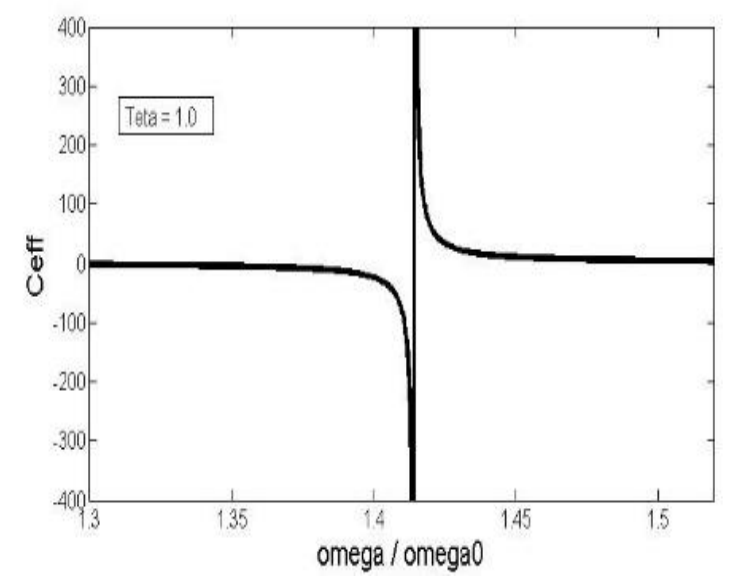

